Running title: Pregnancy and levels of depression and anxiety

\title{
Pregnancy and levels of depression and anxiety: A prospective cohort study of
}

\section{Australian women}

Liana S. Leach $(\mathrm{PhD})^{1^{*}}$ Helen Christensen (Professor) ${ }^{2}$, and Andrew Mackinnon (Professor) $^{3}$

${ }^{1}$ Psychiatric Epidemiology and Social Issues Unit, Centre for Research on Ageing, Health and Wellbeing, The Australian National University, Canberra, ACT, 0200, Australia. Email: Liana.Leach@anu.edu.au

${ }^{2}$ Black Dog Institute, Prince of Wales Hospital, Randwick, NSW, 2031, Australia. Email: h.christensen@blackdog.org.au

${ }^{3}$ ORYGEN Research Centre, The University of Melbourne, VIC, 3052, Australia. Email: Andrew.Mackinnon@unimelb.edu.au

* Corresponding Author: Liana Leach. Centre for Research on Ageing, Health and Wellbeing, Building 62A, The Australian National University, Canberra 0200, Australia. Tel: +61 2 61259725, Fax: +61 2 61251558, Email: Liana.Leach@anu.edu.au 


\begin{abstract}
Objective: The effects of pregnancy on depression and anxiety remain unclear. Previous research is predominantly cross-sectional, not representative of the general community, and does not include data on mental health prior to pregnancy. This study used longitudinal Australian population-based data to examine whether pregnancy is associated with increases in women's anxiety and depression levels (from prepregnancy).
\end{abstract}

Method: A community sample of Australian women aged 20-24 years were recruited prospectively and assessed in 1999, 2003 and 2007. At the follow-up assessments 76 women were pregnant (with no prior children) and 542 remained nulliparous. Mixed Models Repeated Measures Analyses of Variance were undertaken to compare change in levels of anxiety and depression (Goldberg Anxiety and Depression Scales) between those who became pregnant and those who remained non-pregnant.

Results: Pregnancy was not associated with increased symptoms of depression or anxiety. No association was found with depression, while pregnancy was associated with a decrease in anxiety. Including somatic items in the measures of depression and anxiety resulted in higher symptom levels in pregnancy, suggesting possible item bias.

Conclusions: This study is one of the first to follow a community sample of women from pre-pregnancy to pregnancy. The findings suggest that pregnancy is not typically detrimental to women's mental health. The current study offers a starting point for future prospective studies to follow women from pre-pregnancy to postpartum. Study limitations to be improved upon in follow-up research include expanding the sample size, and including both pregnancy specific measures and trimester specific data. Future research should continue to identify those women who are most (and least) at risk during pregnancy in order to target resources and assistance most effectively.

Key words Pregnancy; Antenatal; Anxiety; Depression; Prospective; Longitudinal

\title{
Introduction
}


Depression and anxiety disorders are common during pregnancy and can have serious adverse impacts on women, partners and children (Dunkel-Schetter and Tanner, 2012; Field et al., 2010; Dunkel-Schetter, 2011; Marcus, 2009; Najman et al., 2005). There is little dispute that this is an important issue for women, and that great benefit can be garnered from effective identification, support and treatment. However, a growing focus on mental health during pregnancy, including the introduction of routine screening during pregnancy as part of the National Perinatal Depression Initiative (Yelland et al., 2009), may have promoted the perception that pregnancy is associated with an increase in depression and anxiety. Available epidemiological research suggests this is not necessarily the case. Fundamental questions remain regarding whether pregnancy has positive, negative or neutral effects on psychological wellbeing.

\section{Prevalence of depression and anxiety during pregnancy vs. general population}

If pregnant women are at increased risk of depression and anxiety, the prevalence of these disorders should be higher in pregnant women than the general population of women (Van Bussel et al., 2006). A meta-analysis of 21 studies conducted by Bennett et al. (2004) reported a pooled prevalence for depression of $7.4 \%, 12.8 \%$ and $12.0 \%$, for the first, second and third trimesters of pregnancy respectively. These estimates, which included depression diagnosed by structured interview and symptom scales, are not dissimilar to the $8.7 \%$ rate for 'any prior 12 month affective disorder' reported for women aged 25-34 in the 2007 Australian National Survey of Mental Health and Wellbeing (NSMHWB) (ABS, 2008). Gavin et al. (2005) reported that the prevalence of Major Depression alone was 3.8\%, $4.9 \%$ and $3.1 \%$ by pregnancy trimester (Gavin et al., 2005). 
These rates are again similar to results from the National Comorbidity Survey (NCS) in the US, which reported 5.0\% prevalence of Major Depression for women aged 18-34 (Kessler et al., 2010).

A review by Ross and McLean (2006) found rates of prenatal General Anxiety Disorder (GAD) ranged from 4.4-8.5\% in four separate studies. More recently, Reck et al. (2008) reported that $2.3 \%$ of women experienced GAD between $0-3$ months post-partum. These rates are not dissimilar to 12 month prevalence rates for GAD in the NSMHWB and the NCS; approximately 5\% for women aged 25-34 (Hunt et al., 2002; Wittchen H, 1994).

\section{Comparison studies of pregnant and non-pregnant women}

A number of cohort studies have directly compared the prevalence of depression and anxiety between groups of pregnant and non-pregnant women. O'Hara et al. (1990) found no differences in rates of Major and Minor Depression between pregnant women $(\mathrm{n}=191)$ and a matched group of non-pregnant women $(n=189)$. However, pregnant women were found to have significantly higher levels of sub-threshold depressive symptomatology. Breitkopf et al. (2006) found no difference in anxiety scores between pregnant and nonpregnant women using the State-Trait Anxiety Inventory (STAI). Recent cross-sectional work conducted by Uguz et al. (2010) found comparable rates of mood and anxiety disorders in pregnant women $(8.1 \% ; 15.5 \%)$ and matched controls $(7.5 \% ; 15.0 \%)$. Further cross-sectional work using a nationally representative sample in the US reported that pregnant women had a lower risk of mood disorders than non-pregnant women and no difference in the risk of anxiety disorders (Vesga-Lopez et al., 2008). Taken together, 
these studies give no clear evidence that pregnancy is associated with more disorder. However, the possibility that sub-threshold depression symptoms might be elevated during pregnancy is raised.

\section{Longitudinal, population-based research}

Cross-sectional studies that compare prevalence rates between pregnant women and other women are limited in their ability to quantify the effects of pregnancy on mental health. Meta-analyses and systematic reviews (Bennett et al., 2004; Ross and McLean, 2006) report substantial heterogeneity in the rates of depression and anxiety disorders during pregnancy, likely due to differences in study sample characteristics, methodology, and measurement. In addition, the majority of studies are cross-sectional, and thus, have no prospective information about pre-pregnancy symptoms, or change in symptom levels. The reliability of conclusions is also hampered by the use of convenience samples, often recruited from antenatal clinics.

Mental health changes from pre-pregnancy to pregnancy are best tested using longitudinal, population-level data. By assessing the same sample of women both prior to and during pregnancy, and adopting the same measurement methods at each time point, many of the limitations associated with selection and measurement differences are eliminated. Several studies have measured symptoms throughout pregnancy longitudinally, but did not include a parallel comparison non-pregnant control group or pre-pregnancy mental health status from pregnant participants (Bennett et al., 2004; Figueiredo and Conde, 2011; Martini et al., 2013). Only one identified prospective cohort 
study has investigated whether psychological distress increases from pre-pregnancy to pregnancy. Van Bussel et al. (2006) used a prospective community-based design to assess women in the United Kingdom prior to, during, and after pregnancy. The authors used a general measure of psychological distress (General Health Questionnaire), and found no significant differences in symptom change between the pregnant and control group over time.

\section{Aim}

The current study used three waves of data from a large Australian community sample in which women who later became pregnant were recruited prospectively. We sought to determine whether pregnancy was associated with increases in levels of anxiety and depression, taking into account symptom levels before pregnancy. Depression and anxiety were assessed using a continuous count of symptoms given previous research indicating there may be an increase in subclinical symptom levels (O'Hara et al., 1990; Halbreich, 2004; Lips, 1985).

\section{Method}

\section{Participants and Procedure}

The Personality and Total Health (PATH) Through Life Project is a prospective narrowage cohort community survey concerned with health and well-being (Anstey et al., 2011). The PATH study was approved by the Australian National University's Human Research in Ethics Committee. Three cohorts were recruited from Canberra and Queanbeyan in Australia in 1999 (20-24, 40-44 and 60-64 years). The sample for this study included 
women from the youngest cohort. 1241 women aged 20-24 years were recruited from the Canberra and Queanbeyan electoral roll and completed the baseline assessment in 1999 (58.6\% recruitment rate). Subsequently, $1126(91 \%)$ and $1058(85 \%)$ women completed the first and second follow-up in 2003 and 2007. Only women who participated in at least waves one and two were considered. There were no significant differences in levels of depression or anxiety between women who dropped out before wave 3 and those who remained. Over the 8 years of the study, 76 women were pregnant at follow-up (2003 or 2007 interview). Only primigravidae women were studied as multiple pregnancies would introduce additional confounding factors.

\section{Study Measures}

All measures were consistent across the three waves of data collection. Depression and Anxiety were measured using the Goldberg Depression and Anxiety Scales (Goldberg et al., 1988). Both Scales consist of nine items representing symptoms of depression/anxiety, each rated with a 'yes/no' response (0-9). The Goldberg scales effectively detect elevated levels of depression and anxiety in community samples (Mackinnon et al., 1994), however to our knowledge they have not been used with a pregnant population. Reflecting the approach taken in the Edinburgh postnatal depression scale (Cox et al., 1987), we excluded four somatic items from each scale to reduce the risk of 'pregnancy-bias' (e.g., 'Have you had headaches/neckaches?', 'Have you been sleeping poorly?'). The reduced scales ranged from $0-5$, and demonstrated good reliability within both the pregnant (Cronbach's alpha: depression=.82, anxiety $=.70$ ) and non-pregnant groups (Cronbach's alpha: depression=.81, anxiety=.76). The full Goldberg 
Scales were used in sensitivity analyses. Pregnancy was ascertained by asking the women if they were pregnant, and if so, how many months. Potential confounders adjusted for included age at baseline, years of education, marital status, employment status, recent financial problems, current contraception use, and current medication use for anxiety and/or depression. These confounders were asked at each wave of data collection, and thus were included in the analyses as time-varying covariates.

\section{Cohorts}

The cohort design sought to maximise available data at each wave and to use appropriate groups for comparison (see (Christensen et al., 2010) for a similar approach). Figure 1 shows the relevant patterns in pregnancy identified and the subgroups formed. At wave 1, two groups of women were either pregnant $(n=20)$ or already had children $(n=151)$. Because no information was available about the symptom levels of these women prepregnancy, they were excluded. A further group were excluded as they became pregnant between waves one and two $(n=76)$ (thus there was no information on symptom levels at the time they were pregnancy). Group $1(\mathrm{G} 1, \mathrm{n}=30)$ consisted of women who were pregnant at wave 2. To assess change in anxiety and depression levels in across waves 1 and 2, Group 1 was compared with Group 2 (G2, n=700), who remained never pregnant at both time points. Group $3(\mathrm{G} 3, \mathrm{n}=46)$ consisted of those who were pregnant at wave three. To assess change in anxiety and depression levels in across waves 2 and 3, Group 3 was compared with Group $4(\mathrm{G} 4, \mathrm{n}=542)$ who remained never pregnant at both time points. 
Insert Figure 1 about here

\section{Statistical Analyses}

Independent samples t-tests and chi-square tests were used to test baseline differences. Mixed Model Repeated Measures (MMRM) ANOVAs with pregnancy group and wave as factors assessed differences in change of levels of anxiety and depression between the relevant subgroups. The covariates were included as time-varying factors to adjust for changes in external correlates over time. Within-participant variation was modelled using an unstructured covariance matrix. The outcomes of conventional tests of each main effect and the interaction were not relevant to this study. Instead, a two degree of freedom contrast was specified to test the two comparisons of interest: 1) Change from wave one to two was compared between those pregnant at wave two (G1) and those not pregnant at or before wave two (G2) (Comparison 1), and 2) Change from wave two to wave three was compared between those pregnant at wave three (G3) and those not pregnant at or before wave three (G4) (Comparison 2). Results from each individual comparison were available, enabling exploration of effects present only for wave two or for wave three. The results from each individual comparison were also pooled to provide an aggregate (and more powerful) estimate of overall change.

Sensitivity analyses indicated that, given the size of relevant subgroups, individual contrasts would have $80 \%$ power to detect between-group differences in the range 0.3 to 0.5 standard deviations. Significance was set at $p=0.05$. 


\section{Results}

Table 1 shows baseline information for each sample group included in each comparison.

G1 was older $(t(728)=-2.99, p=.003)$ and more likely to be married $\left(\chi^{2}=35.88(1), p<.001\right)$ than G2. G3 was no different on any of the covariates to G4. Additional comparisons between the pregnant G1 and G3, showed G1 was older than G2 $(t(74)=-2.78, p=.007)$ and more likely to be married $\left(\chi^{2}=16.37(1), p<.001\right)$.

Insert Table 1 about here

Table 2 shows the mean scores for levels of depression and anxiety at each wave. Most importantly, at wave one (baseline) there were no significant differences between any of the groups, for either depression or anxiety levels. At wave two G1 had lower anxiety levels than $\mathrm{G} 2(t(272)=-2.19, p=.029)$, but there were no differences between G3 and G4. At wave three, there were no differences between any of the groups.

Insert Table 2 about here

The hypothesis testing contrasts within the MMRM ANOVAs found no significant differences in change from waves one to two (Comparison 1), or waves two to three (Comparison 2), between those who became pregnant and those who remained nonpregnant for depression (see Table 3). Thus, the aggregate effect of both contrasts combined was not significant $(F(2,781)=1.25, p=.288)$. Changes in anxiety levels were significantly different from waves one and two between those who became pregnant and 
those who did not (Comparison 1). Those who became pregnant at wave two experienced a greater decrease in anxiety symptoms (from pre-pregnancy) than those who remained non-pregnant (mean difference in change scores $=-.952, p=.009$ ). While change in anxiety levels between waves two and three showed a similar trend, the difference was not significant (Comparison 2). However, the aggregate effect of both contrasts combined was significant $(F(2,775)=4.16, p=.016)$. The Estimated Marginal Mean scores for depression and anxiety symptoms for each sample group across both comparisons can be seen in Figure 2 (adjusted for covariates).

Insert Table 3 about here

Insert Figure 2 about here

Sensitivity analyses

The MMRM ANOVAs were repeated using the full Goldberg Depression and Anxiety Scales to investigate the implications of excluding the somatic items. Again, there were no significant differences in change from waves one to two between those who became pregnant and those who did not for depression (Comparison 1); however, there were significant differences between waves two and three (Comparison 2). Those who became pregnant at wave three experienced a greater increase in depression scores (from prepregnancy) than those who remained non-pregnant $(t(680)=2.13, p=.033)$. However, the 
aggregate effect of the two contrasts was not significant $(F(2,781)=2.36, p=.095)$. When anxiety symptoms were examined the significant effects from the previous analyses using the restricted scales were no longer present. This was the case both for Comparison 1 assessing change from waves one to two $(t(751)=-1.65, p=.100)$, and the aggregate across both comparisons $(F(2,764)=1.38, p=.252)$.

\section{Discussion}

Pregnancy was not found to be associated with increases in depression or anxiety. There was no association between becoming pregnant and change in depression. Pregnant women experienced a greater decline in anxiety from pre-pregnancy levels than those who remained non-pregnant. Importantly, these effects are unlikely to be the result of preexisting differences in psychological distress, or simply an artefact involving regression to the mean, as the groups did not differ in levels of depression or anxiety pre-pregnancy. Including somatic items resulted in greater anxiety and depression for women whilst pregnant, indicating possible item bias.

While much of the previous literature has been discordant, the current findings are consistent with the only other prospective cohort study with pre-pregnancy data available. This study also found no change in psychological distress during pregnancy (Van Bussel et al., 2006) using the General Health Questionnaire as an outcome measure (which does not contain items assessing somatic symptoms). We know of no other study reporting a decrease of anxiety symptoms from pre-pregnancy to pregnancy. We expected that pregnancy might lead to small but significant sub-threshold symptom increases in depression, but this was not the case. This contrasts with O'Hara et al. (1990) and 
Halbreich (2004) who suggest pregnancy may be associated with higher levels of symptomatology but not diagnosed disorders. However, a closer examination of O’Hara et al.'s study shows that pregnant women only reported increased depression when depression was measured using both the Cognitive-Affective and the Somatic subscales of the Beck Depression Inventory. Consistent with the current study's findings, there were no significant differences between pregnant and non-pregnant groups on the Cognitive-Affective subscale alone.

\section{Measurement implications}

The current study clearly demonstrates that the assessment tool used to measure depression and anxiety during pregnancy influences the estimates obtained. Our findings indicate that measures that include somatic items yield higher estimates of morbidity amongst pregnant women than measures which exclude these items. While the current study did not conduct a formal analysis of item bias with regard to pregnancy status, the findings concord with previous research suggesting somatic items can be 'pregnancybiased' (O'Hara et al., 1990; Matthey, 2010). While the concern around 'perinatal-biased' measures is not new, and was the impetus for the development of the Edinburgh Post Natal Depression Scale (Cox et al., 1987; Pop et al., 1992), this remains a controversial issue. Debate persists about whether the DSM diagnostic criteria for depression in the perinatal period should include symptoms 'normal' during pregnancy and new parenthood, such as 'weight or appetite change', 'sleep disturbance' and 'fatigue', as is currently the case (Matthey, 2010). 


\section{Limitations}

Several limitations to the current study need to be noted. Variation in anxiety across specific time-points within pregnancy (e.g. by trimester) could not be confidently assessed due to the sample size available. Related to this point, data for individuals in the PATH study was collected every 4 years. Although several time-varying variables were adjusted for, other (unmeasured) life circumstances impacting on mental health may have intervened in the interim between collecting baseline data and follow-up pregnancy. The experience of pregnancy was not assessed beyond the age of 32, as the PATH cohort was aged 24-28 at wave 2 and 28-32 at wave 3. While this age-range is likely to have captured many women's first births, (the median age of first-time mothers in Australia was 29.1 in 2012 (ABS, 2012)), it may be that women older than 32 have a different experience of their first pregnancy. Diagnostic measures of mental health (i.e., mental disorders) and measures specifically designed for the perinatal period were not available. However, the primary concern with general measures is the inclusion of items measuring somatic symptoms; and the potential impact of these items was assessed in the current study.

The current study's ability to detect change was potentially limited because of the size of the pregnant groups (aggregate $n=76$ ). As such, steps were taken to maximise power and minimise false negative findings, including no adjustment for multiple testing and aggregate testing of contrasts. Small effects were found to be significant at the .05 level adopted, as predicted in preliminary power analyses. The small sample size also limits 
generalizability of the findings (as discussed further below), and highlights the need for future follow-up research.

It is important to note the population used in the current study, and how that might impact the findings. The sample was taken from Canberra (ACT) and Queanbeyan in Australia. This location has been shown to be more affluent with lower unemployment rates than other states and territories in Australia (ABS, 2014), suggesting the findings may not be representative of the broader Australian population. However, in defence of the population used, findings from the beyondblue National Post-natal Depression Program, did not find lower rates of post-natal depression in the ACT (EPDS >9 16\%; EPDS >12 7.1\%) as opposed to Australian rates (EPDS >9 15.5\%; EPDS >12 7.5\% (Buist et al., 2008)). In any case, further research replicating the current findings is needed. Ideally, this research should include representatives from sub-populations where psychological problems may be most prevalent, such as non-Caucasian, unmarried, those with marital problems and teenage individuals (Bilszta et al., 2008; Halbreich, 2004; Quinlivan et al., 2004; Rich-Edwards et al., 2006). Data on other potential risk factors should also be included, such as women's pregnancy circumstances (e.g., unwanted/unintended pregnancy) (Fergusson et al., 2008; Dingle et al., 2008). While the aim of the current study was to take a broad population-based approach to consider whether women in the community 'generally' experience increases in anxiety and depression during pregnancy, further research is needed to tease out how the current findings might differ for different types of women and build on the body of 'risk factor' research available. 
While there are limitations to the current study, it is important to also recognise its methodological advances. The availability of longitudinal data and the use of longitudinal analysis techniques, together with information on pre-pregnancy symptomology is unique, and provides a framework for future studies to advance upon.

\section{Conclusions}

This study is one of the first to follow a community sample of women from prepregnancy, and assess the impact of pregnancy on levels of depression and anxiety. The results show no increase in depression or anxiety symptoms during pregnancy. To date, the rhetoric has largely focused on the negative psychological symptoms associated with pregnancy. This is understandable, given the adverse impacts of pre- and post-natal depressive and anxiety disorders for women themselves, their partners and children. While the current study suggests that the average pregnant women is not at increased risk, it is important to remember that some pregnant women will be at greater risk, and future risk-factor research should continue to examine how we can best identify and offer treatment. Discussion should continue regarding whether routine and universal screening, as recommended by the National Perinatal Depression Initiative, is the most effective way to identify women most at risk.

\section{Acknowledgements}

The PATH Through Life Project is funded by the Australian National Health and Medical Research Council (NHMRC) Grant number 418039. H.C. is funded by NHMRC Fellowship number 525411. L.L is funded by NHMRC Fellowship number 1035803. The 
NHMRC had no further role in study design; in the collection, analysis and interpretation of data; in the writing of the report; and in the decision to submit the paper for publication. The authors would like to thank T. Slade for feedback on the methodology, analyses and final draft of the paper. In addition, the authors would like to thank K. J. Anstey, A. F. Jorm, P. J. Butterworth, P. A. Jacomb and K. Maxwell for contributions to the design of the PATH Through Life Project and the PATH project participants.

\section{Declaration of Conflicting Interests}

The Author(s) declare(s) that there is no conflict of interest. 


\section{References}

ABS. (2008) National Survey of Mental health and Wellbeing: Summary of results, 2007. Cat. No. 4326.0. Canberra: Australian Bureau of Statistics.

ABS. (2012) Births. Cat. No. 3301.0. Canberra: Australian Bureau of Statistics.

ABS. (2014) Labour Force Australia. Cat. No. 6202.0. Canberra: Australian Bureau of Statistics.

Anstey KJ, Christensen H, Butterworth P, et al. (2011) Cohort Profile: The PATH through life project. International Journal of Epidemiology.

Bennett HA, Einarson A, Taddio A, et al. (2004) Prevalence of depression during pregnancy: systematic review. Obstetrics and Gynecology 103: 698-709.

Bilszta JL, Tang M, Meyer D, et al. (2008) Single motherhood versus poor partner relationship: outcomes for antenatal mental health. The Australian and New Zealand Journal of Psychiatry 42: 56-65.

Breitkopf CR, Primeau LA, Levine RE, et al. (2006) Anxiety symptoms during pregnancy and postpartum. Journal of Psychosomatic Obstetrics \& Gynecology 27: 157-162.

Buist AE, Austin MP, Hayes BA, et al. (2008) Postnatal mental health of women giving birth in Australia 2002-2004: findings from the beyondblue National Postnatal Depression Program. Aust N Z J Psychiatry 42: 66-73.

Christensen H, Leach LS and Mackinnon A. (2010) Cognition in pregnancy and motherhood: prospective cohort study. The British Journal of Psychiatry 196: 126-132.

Cox JL, Holden JM and Sagovsky R. (1987) Detection of Postnatal Depression: Development of the 10-item Edinburgh Postnatal Depression scale. British Journal of Psychiatry 150: 782-786.

Dingle K, Alati R, Clavarino A, et al. (2008) Pregnancy loss and psychiatric disorders in young women: an Australian birth cohort study. British Journal of Psychiatry 193: 455-460.

Dunkel-Schetter C. (2011) Psychological science on pregnancy: stress processes, biopsychosocial models, and emerging research issues. Annual Review of Clinical Psychology 62: 531-558.

Dunkel-Schetter C and Tanner L. (2012) Anxiety, depression and stress in pregnancy: implications for mothers, children, research, and practice. Current Opinion in Psychiatry 25: 141-148.

Fergusson DM, Horwood LJ and Boden JM. (2008) Abortion and mental health disorders: evidence from a 30-year longitudinal study. British Jounral of Psychiatry 193: 444-451.

Field T, Diego M and Hernandez-Reif M. (2010) Prenatal depression effects and interventions: a review. Infant Behavior and Development 33: 409-418.

Figueiredo B and Conde A. (2011) Anxiety and depression in women and men from early pregnancy to 3-months postpartum. Arch Womens Ment Health 14: 247-255.

Gavin NI, Gaynes BN, Lohr KN, et al. (2005) Perinatal Depression: A Systematic Review of Prevalence and Incidence. Obstetrics \& Gynecology 106: 1071-1083 1010.1097/1001.AOG.0000183597.0000131630.db. 
Goldberg D, Bridges K, Duncan-Jones P, et al. (1988) Detecting anxiety and depression in general medical settings. British Medical Journal 297: 897-899.

Halbreich U. (2004) Prevalence of mood symptoms and depressions during pregnancy: implications for clinical practice and research. CNS Spectrums 9: 177-184.

Hunt C, Issakidis C and Andrews G. (2002) DSM-IV generalized anxiety disorder in the Australian National Survey of Mental Health and Well-Being. Psychological Medicine 32: 649-659.

Kessler RC, Birnbaum H, Bromet E, et al. (2010) Age differences in major depression: results from the National Comorbidity Survey Replication (NCS-R). Psychological Medicine 40: 225-237.

Lips HM. (1985) A longitudinal study of the reporting of emotional and somatic symptoms during and after pregnancy. Social Science and Medicine 21: 631-640.

Mackinnon A, Christensen H, Jorm AF, et al. (1994) A latent trait analysis of an inventory designed to detect symptoms of anxiety and depression using an elderly community sample. Psychological Medicine 24: 977-986.

Marcus SM. (2009) Depression during pregnancy: rates, risks and consequences-Motherisk Update 2008. Canadian Journal of Clinical Pharmacology 16: e15-22.

Martini J, Wittich J, Petzoldt J, et al. (2013) Maternal anxiety disorders prior to conception, psychopathology during pregnancy and early infants' development: a prospective-longitudinal study. Arch Womens Ment Health 16: 549-560.

Matthey S. (2010) Are we overpathologising motherhood? Journal of Affect Disorders 120: 263-266.

Najman JM, Hallam D, Bor WB, et al. (2005) Predictors of depression in very young children--a prospective study. Social Psychiatry and Psychiatric Epidemiology 40: 367-374.

O'Hara MW, Zekoski EM, Philipps LH, et al. (1990) Controlled prospective study of postpartum mood disorders: comparison of childbearing and nonchildbearing women. Journal of Abnormal Psychology 99: 3-15.

Pop VJ, Komproe IH and van Son MJ. (1992) Characteristics of the Edinburgh post natal depression scale in The Netherlands. Journal of affective disorders 26: 105-110.

Quinlivan JA, Tan LH, Steele A, et al. (2004) Impact of demographic factors, early family relationships and depressive symptomatology in teenage pregnancy. Australia and New Zealand Journal of Psychiatry 38: 197-203.

Rich-Edwards JW, Kleinman K, Abrams A, et al. (2006) Sociodemographic predictors of antenatal and postpartum depressive symptoms among women in a medical group practice. Journal of Epidemiology and Community Health 60: 221-227.

Ross LE and McLean LM. (2006) Anxiety Disorders During Pregnancy and the Postpartum Period: A Systematic Review. Journal of Clinical Psychology 67: 1285-1298.

Van Bussel JCH, Spitz B and Demyttenaere K. (2006) Women's Mental Health Before, During, and After Pregnancy: A Population-Based Controlled Cohort Study. Birth 33: 297-302.

Vesga-Lopez O, Blanco C, Keyes K, et al. (2008) Psychiatric Disorders in Pregnant and Postpartum Women in the United States. Archives of General Psychiatry 65: 805815. 
Wittchen H ZS, Kessler RC, Eaton WW. (1994) Dsm-III-R generalized anxiety disorder in the national comorbidity survey. Archives of General Psychiatry 51: 355-364.

Yelland JS, Sutherland GA, Wiebe JL, et al. (2009) A national approach to perinatal mental health in Australia: exercising caution in the roll-out of a public health initiative. Medical Journal of Australia 191: 276-279. 


\section{Tables}

Table 1: Socio-demographics and other covariate information at baseline (Wave 1).

\begin{tabular}{lllll}
\hline & \multicolumn{2}{c}{ Comparison 1 (Wave1-Wave2) } & \multicolumn{2}{l}{ Comparison 2 (Wave2-Wave3) } \\
\hline & $\begin{array}{l}\text { Pregnant W2 } \\
\text { Group 1 }\end{array}$ & $\begin{array}{l}\text { Never Pregnant } \\
\text { Group 2 }\end{array}$ & $\begin{array}{l}\text { Pregnant W3 } \\
\text { Group 3 }\end{array}$ & $\begin{array}{l}\text { Never Pregnant } \\
\text { Group 4 }\end{array}$ \\
\hline $\mathrm{N}$ & 30 & 700 & 46 & 542 \\
Age & $23.33(1.49)$ & $22.51(1.48)$ & $22.35(1.52)$ & $22.46(1.47)$ \\
Married/de facto (\%) & $56.7 \%$ & $15.0 \%$ & $13.0 \%$ & $11.6 \%$ \\
Education (years) & $15.15(1.43)$ & $15.04(1.39)$ & $15.20(1.42)$ & $14.99(1.38)$ \\
Employed (\%) & $96.7 \%$ & $90.3 \%$ & $97.8 \%$ & $88.6 \%$ \\
Financial strain (\%) & $26.7 \%$ & $23.1 \%$ & $26.1 \%$ & $23.4 \%$ \\
Contraception use (\%) & $72.4 \%$ & $55.9 \%$ & $65.2 \%$ & $54.4 \%$ \\
Depression med (\%) & $3.3 \%$ & $6.9 \%$ & $2.2 \%$ & $7.6 \%$ \\
Anxiety med (\%) & $6.7 \%$ & $8.0 \%$ & $4.3 \%$ & $7.9 \%$ \\
\hline
\end{tabular}

Notes: ${ }^{a}$ Group 4 is a subset of Group 2. 
Table 2: Means and standard deviations for pregnant and non-pregnant comparison groups at each wave.

Comparison 1 (Wave1-Wave2) Comparison 2 (Wave2-Wave3)

\begin{tabular}{|c|c|c|c|c|}
\hline & $\begin{array}{l}\text { Pregnant W2 } \\
\text { Group } 1\end{array}$ & $\begin{array}{l}\text { Never Pregnant } \\
\text { Group } 2\end{array}$ & $\begin{array}{l}\text { Pregnant W3 } \\
\text { Group } 3\end{array}$ & $\begin{array}{l}\text { Never Pregnant } \\
\text { Group } 4^{\mathrm{a}}\end{array}$ \\
\hline \multicolumn{5}{|l|}{ Wave 1} \\
\hline Anxiety (0-5) & $2.63(1.69)$ & $2.58(1.71)$ & $2.24(1.73)$ & $2.62(1.70)$ \\
\hline Depression (0-5) & $1.57(1.83)$ & $1.80(1.65)$ & $1.35(1.49)$ & $1.83(1.64)$ \\
\hline \multicolumn{5}{|l|}{ Wave 2} \\
\hline Anxiety (0-5) & $1.72(1.56)$ & $2.43(1.69)$ & $2.35(1.62)$ & $2.41(1.72)$ \\
\hline Depression (0-5) & $1.55(1.35)$ & $1.70(1.35)$ & $1.41(1.59)$ & $1.80(1.74)$ \\
\hline \multicolumn{5}{|l|}{ Wave 3} \\
\hline Anxiety (0-5) & - & - & $2.04(1.66)$ & $2.42(1.76)$ \\
\hline Depression (0-5) & - & - & $1.84(1.66)$ & $1.70(1.70)$ \\
\hline
\end{tabular}

Notes: ${ }^{a}$ Group 4 is a subset of Group 2. Scores for groups not included in the contrasts for wave 3 are indicated by - .

Table 3. Mean differences in change scores for anxiety and depression by pregnancy status for each wave.

\begin{tabular}{|c|c|c|c|c|c|}
\hline & \multicolumn{2}{|c|}{ Comparison 1 (W1-W2) } & \multicolumn{2}{|c|}{ Comparison 2 (W2-W3) } & \multirow[t]{2}{*}{ Aggregated } \\
\hline & $\begin{array}{l}\text { Value } \dagger \\
(95 \% \text { CI })\end{array}$ & $\mathrm{p}$ & $\begin{array}{l}\text { Value } \dagger \\
(95 \% \text { CI) }\end{array}$ & $\mathrm{p}$ & \\
\hline Anxiety & $\begin{array}{l}-0.952 \\
(-1.67-.233)\end{array}$ & 0.009 & $\begin{array}{l}-.434 \\
(-.991-.122)\end{array}$ & 0.126 & 0.016 \\
\hline Depression & $\begin{array}{l}-0.014 \\
(-.699-.671)\end{array}$ & 0.968 & $\begin{array}{l}.434 \\
(-.111-.980)\end{array}$ & 0.118 & 0.288 \\
\hline
\end{tabular}

Notes: $†$ The value of the contrast is the mean difference in change from one wave to the next between the pregnant group(s) and the non-pregnant group(s). $\dagger$ is adjusted for age, education, marital status, employment, financial problems, using contraception, depression and anxiety medication. 


\section{Figures}

Figure 1. Cohort groups defined by pregnancy status at each wave.

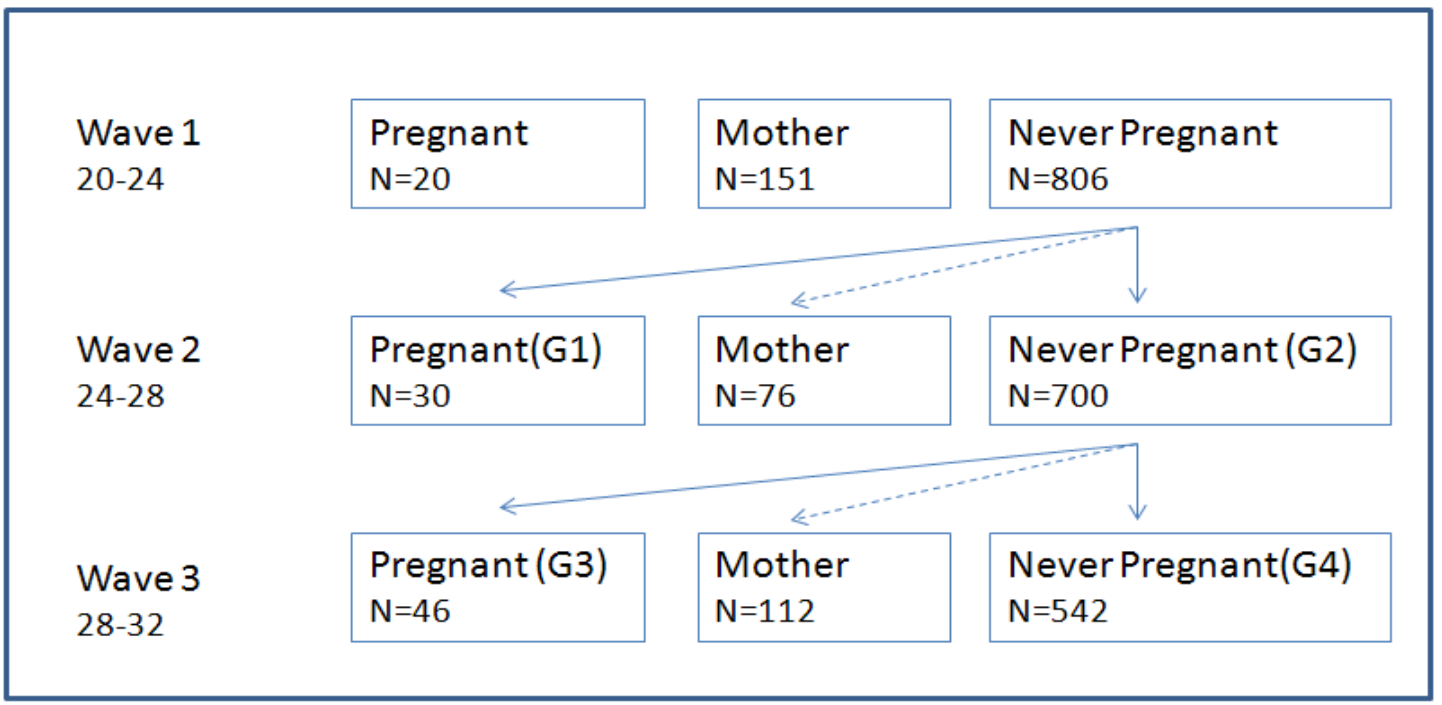

Notes: Additional groups not shown in the figure: 115 women who dropped out post wave one and 149 women who experienced multiple pregnancies between waves of data collection. 
Figure 2. Change in anxiety and depression by pregnancy group.

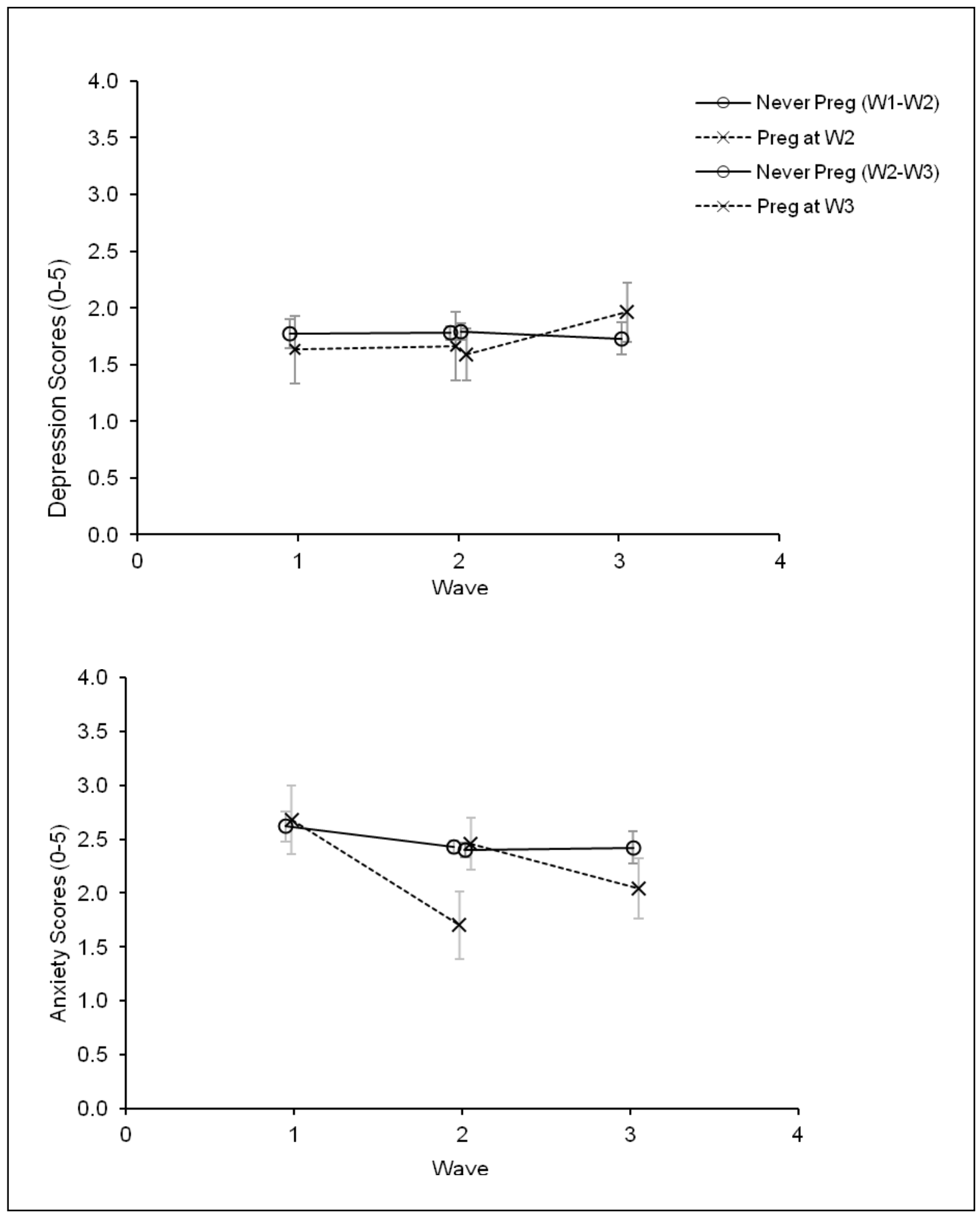

Notes: Estimated Marginal Means adjusted for time-varying covariates age, education, marital status, employment, financial problems, using contraception, depression and anxiety medication. 